\title{
COMMENTARY
}

\section{Achieving Universal Access to Health Care in Africa: The Role of Primary Health Care}

\author{
Andrew G. Onokerhoraye \\ Professor Emeritus, University of Benin, Benin City, Nigeria and Executive Director, Centre for Population and Environmental \\ Development (CPED), Benin City, Nigeria
}

*For Correspondence: Email: agonoks@yahoo.com; Phone: +2348023346647

Following the expiration of the eight Millennium Development Goals (MDGs) that were used as frameworks for shaping progress in development between 2000 and 2015, seventeen new Sustainable Development Goals (SDGs) were adopted by governments at the UN General Assembly in September, 2015. SDG3 focuses on health i.e. to "Ensure healthy lives and promote well-being for all at all ages". This goal is translated into 13 targets: three focus on reproductive and child health; three concern communicable diseases, non-communicable diseases, and addiction; two focus on environmental health; one relates to achieving universal health coverage (UHC); while four targets relate to tobacco control, vaccines and medicines, health financing and workforce, and global health risk preparedness. This brief commentary examines how universal health coverage (UHC) in African countries can be achieved through the strengthening of the primary health care system ${ }^{1}$.

Between $6^{\text {th }}$ and $12^{\text {th }}$ September 1978 , at an International Conference on Primary Health Care, in Alma Ata, Kazakhstan, USSR, 134 countries endorsed the 'health for all' programme through the Alma Ata Declaration ${ }^{2}$. The programme was to be driven by the Primary Health Care (PHC) system. The PHC system has been the platform through which many African countries have committed themselves to providing their citizens with provision of equitable access to health care. The PHC concept stands on five key principles, designed to work together and be implemented simultaneously to bring about better outcomes for the entire population. These are: (i) Accessibility (equal distribution): this is the first and most important key to PHC. Health care services must be equally shared by all the people irrespective of race, creed or economic status. This concept helps to shift the accessibility of healthcare from the cities to the rural areas where the most needy and vulnerable groups of the population live; (ii) Health promotion: involves all the important issues of health education, nutrition, sanitation, maternal and child health, and prevention and control of endemic diseases. Through health promotion, individuals and families build an understanding of the determinants of health and develop skills to improve and maintain their health and wellbeing; (iii) Appropriate technology: technology that is scientifically sound, adaptable to local needs, and acceptable to those who apply it and for whom it is used; (iv) Inter-sectoral collaboration: to be able to improve the health of local people the PHC programme needs not only the health sector, but also the involvement of other sectors, like agriculture, education and housing; and (v) Community participation: this includes meaningful involvement of the community in planning, implementing and maintaining their health services ${ }^{3}$. Through the involvement of the community, maximum utilisation of local resources, such as manpower, money and materials, can be utilised to fulfil the goals of PHC.

The key health challenges articulated in the SDG3, especially those which focus on reproductive and child health, communicable diseases, chronic illnesses, addiction, and environmental health problems can be addressed through a person-centred and population-based approach to primary health care. Well integrated primary health care system is essential for the achievement of UHC both equitably and costeffectively. At the same time, an effective primary health care system can contribute to the achievement of many of the other 16 SDGs. 
Primary care teams in Africa can through the discharge of their main responsibilities make contributions across the SDGs. For example, primary health care can contribute to ending extreme poverty, improve nutrition, provide health education and promote lifelong learning. Furthermore, primary health care has major roles to play in the empowerment of individuals and communities so as to reduce inequities and promote justice, enable access to safe water and sanitation, encourage productive and sustainable employment, advocate for healthy and sustainable living environments, and at the same time promote peaceful communities.

Despite the key role which primary health care can play in the attainment of SDGs, the promotion of an effective and efficient primary health care system remains elusive to many governments, policy makers, funders, and healthcare providers in many developing countries in general and in African countries in particular. Thus, nearly 40 years after the Alma-Ata declaration, the absence of a reference to primary health care in the SDGs and their targets is a major oversight. One major factor responsible for the failure of primary health care in many countries in Africa since the Alma-Ata declaration is the lack of effective strategies for implementation. Critical frameworks for monitoring and assessing the implementation of PHC activities are also lacking. In view of the fact that many African governments have adopted PHCs as frameworks for developing their health care systems, approaches for accountability and scale-up would need to be identified.

PHC is a critical issue that needs to be addressed by health policy makers and practitioners in African countries as they develop implementation strategies for the SDGs. If effective and tested strategies are not articulated on how primary health care systems should function, there is high probability that the failures of the past which tended to weaken the contributions of primary health care services to the health care system will be repeated in the implementation of the SDGs. It is essential that governments and other stakeholders in African countries are ambitious in measuring progress towards the delivery of primary health care that will address the SDGs. This monitoring includes the use of indicators that capture the principles of equity, community participation, prevention, appropriate technology, and inter-sectoral collaboration in the implementation of PHCs in African countries. There is also the need to document elements related to first contact, continuity, comprehensiveness, coordination, and family and community orientation which, evidence suggests, make primary care services successful. Health financing indicators need to track government expenditure in this area and provide information on the economic accessibility of primary care services.

Key primary health care issues that should be monitored and strengthened include the following: (i) Location of Primary Health Care Centres: It's essential to measure where primary care centres are based in relation to the populations they serve. If half the population of a country live in rural areas, then the majority of health services and human resources for health should also be located in rural areas. In particular, we need to measure whether primary health care centres are effectively supporting those populations in greatest need, including the poor and disadvantaged; (ii) Size and Training of Health Workforce: There is need to regularly evaluate the numbers of primary care nurses, midwives, birth attendants and community health workers available to meet the needs of local populations. In addition, there is need to measure the percentage of graduates from professional health training institutions who are expected to work in community-based health services. We know that many countries in Africa are training too many hospital-based medical specialists at the expense of strengthening their primary care medical workforce. This leads to health services not being available in the communities where they are mostly needed; (iii) Value placed on Primary Care Providers: Developing measures of whether a country values its health care professionals who work in rural communities is essential. For example, do nurses and doctors working in primary care, especially in remote rural areas receive higher incomes than nurses and doctors working in hospitals in big cities? If not what can be done to reverse the situation? and (iv) Patient 


\section{Andrew G. Onokerhoraye}

and Health Worker Interactions: One of the great challenges to achieving health care for everyone is ensuring services are focused on the entirety of an individual's health needs, with attention to how and when they need to access them. This can be measured through indicators such as patient satisfaction; access to care after hours, home-based care, interpreters, disability-friendly services; and the integration of preventive care and health promotion into primary care services ${ }^{5}$.

An effective and sustainable PHC system in developing countries, such as those in Africa, is expensive in terms of material and human resources. Nevertheless, the PHC system provides a more holistic approach to addressing the health needs of individuals especially those located in remote communities, promotes the development of the overall health infrastructure and is critical for sustained improvements in the health of deprived communities $^{6}$. It is in this context that PHC services are indispensable components to the success of the national health systems in African countries and the attainment of the SDGs. Revitalizing PHC principles without developing a framework to address concrete measures for health improvements is not sufficient. To do this, particularly in a time of financial and security challenges which African countries are facing at the moment, requires a willingness to dialogue and appreciate a range of different views working toward a consensus. With political commitment, an enabling economic environment and equitable
Achieving Universal Access to Health Care in Africa

distribution of resources, comprehensive $\mathrm{PHC}$ has proved to be a better strategy in achieving the goal of health for all. Finally, countries in Africa need strong political will, sound economic policies, and coordinated international efforts to achieve the health care components of the SDGs.

\section{Conflict of Interest: None}

\section{References}

1. Luisa M Pettigrew, Jan De Maeseneer, Maria-Inez Padula Anderson, Akye Essuman, Michael R. Kidd, and Andy Haine, The Lancet on November 28, 2015. World Health Organisation (WHO) (1978), Declaration of Alma Ata, http://www.who.int/hpr /NPH/declaration_almaata.pdf.

2. Omuta, Gideon E.D., Andrew G. Onokerhoraye and Francis A. Onojeta, Infrastructure and PHC Services in the Niger Delta Region of Nigeria: The Case of Delta State; BahnhofstraBe, Germany: LAP LAMBERT Academic Publishing, 2016.

3. Abdulrahhem, I.S., Oladipo, A.R. and Amodu, M.O. (2012), "Primary Health Care Services in Nigeria: Critical Issues and Strategies for Enhancing the use by Rural Communities", Journal of Public Health and Epidemiology, Vol. 4, No. 1,(January), pp.5-12.

4. Michael R. Kidd Achieving Global Health Goals by Strengthening Primary Health Care, Huffington Post October 27, 2015.

5. Omuta, GE.D, Onokerhoraye, A.G., Okonofua, F., Obanovwe, G., Isah, E., Chiwuzie, J., Okoro, F., Onojeta, F., Omoraka, F., Eregare, J. and Akpomera, E. (2014), "Perspectives on Primary Health Care in Nigeria: past Present and Future",CPED Monograph Series No 10; TTI/IDRC, 76 pp. 